\title{
Stochastic cycles for modelling workload distributions in military headquarters
}

\author{
$\underline{\text { A.C. Kalloniatis }}^{\text {a }}$, I.D.G. Macleod ${ }^{a}$ and P. La ${ }^{a}$ \\ ${ }^{a}$ Joint Operations Division, Defence Science and Technology Organisation, Canberra, ACT, 2600 \\ Email: alexander.kalloniatis@dsto.defence.gov.au
}

\begin{abstract}
Military headquarters coordinate planning and monitoring of military task forces in support of an overall commander. Despite advances in technology, these organisations remain human intensive. Adequately staffing a headquarters is challenging in resource constrained environments where senior decision-makers must also consider the manpower needs of deployed task forces together with their training and sustainment. At MODSIM09 we presented a high level workload model based on competition between irregular linear business processes (triggered by unpredictable external events) and regular cyclic routines that follow a headquarters 'Battle Rhythm'. We extend this representation here by modelling a richer spectrum of activities to higher fidelity and unifying the treatment of their timing by spanning the range between irregular and regular. We call this the 'Stochastic Cycles' approach and demonstrate its utility by modelling planning in a hypothetical headquarters, where there are concurrent strategic, operational and logistic considerations. Using Discrete Event Simulations we show how data on Tasks, Roles and Staff for an existing headquarters enable decision-makers to explore the effect of alternative staff allocations. We discuss the advantages and disadvantages of the Stochastic Cycles approach in organisational modelling.
\end{abstract}

Keywords: organisations, military, planning, monitoring, stochastic, simulation 


\section{INTRODUCTION}

Military Headquarters are organisations staffed to support the Commander of a military force in planning of operations and in monitoring and controlling execution of those operations by the force. A headquarters encompasses diverse specialists, in areas including logistics, intelligence and Information and Communications Technology (ICT) systems. The specialists work with strategists and planners to develop integrated plans for military operations in response to unforeseen events in the dynamical external environment. Such plans seek to fulfil the Commander's Intent, which in turn (in western democratic nations) is subordinate to the intent of elected governments. Known as Joint Planning, military doctrine usually provides a Business Process for this integration of higher Intent, specialist input and innovative strategic design into a plan. To the extent that military operations lie in the domain of Wicked Problems (Rittel and Webber, 1973), in practice planners diverge in apparently random ways from the linear sequence of a Business Process. In this era of high tempo military operations together with constrained numbers of personnel available for deployment in Defence Forces, modelling and simulation is proving invaluable as an input to decisions about staff numbers in a military headquarters. We describe an innovative and practical method of providing such modelling and simulation, using randomness of staff work as a defining feature.

We have been involved over the years in modelling aspects of such organisations at varying levels of fidelity. Originally this was through traditional Business Process Modelling, which, as described for example by Aguilar-Savén (2004), focuses on ordered sequences of activities for the development of a product. Simulation of multiple business processes competing for resources such as staff is one means of testing the compatibility of such processes with staff structures and numbers. Except in crisis situations, military planning typically can have lead times of several days to months and is often modelled in terms of a process. In analysing how headquarters monitor and control the execution of their operations (subsequent to planning), we confronted highly reactive and indecomposable activities. Military staff seek to regulate this using 'Battle-Rhythm', and this pattern offered the clue to model associated workloads in terms of 'competing cycles' (Kalloniatis et al., 2009).. Planning in the context of ongoing operations therefore exhibits similar reactive and chaotic behaviours, which makes them 'Complex Endeavours' as recognised in Alberts and Hayes (2007). However, the Wicked Problem dimension means that planning well in advance of 'boots hitting the ground' exhibits both regular (process-driven) and irregular features. We demonstrate a new modelling representation of headquarters work that incorporates both stochastic and cyclic triggering of activities by which staff are allocated to planning. In this respect the model is a closer approach to reality than business process representations but at the same time is more data efficient than 'complexity' motivated agent-based distillations. Our model allows decision-makers to be informed on the appropriateness of staff numbers and disposition in a headquarters design.

In the next section we detail the nature of joint military planning. We then outline our new model and hypothetical data. Simulation results for different distributions of staff are then presented. We conclude by discussing the benefits and costs associated with our new approach and its scope for refinement.

\section{MODELLING MILITARY PLANNING}

Planning, according to Mintzberg (1994), is "a formalised procedure to produce an articulated result, in the form of an integrated system of decisions". Mintzberg emphasises that real planning is organic and ad hoc. As a formalised process in the military context, planning is conducted using the Military Appreciation Process (MAP) in the Australian Army and the Operational Planning Process (OPP) in the United States. Guitouni et al. (2006) present a summary of such processes when used for likely or certain future events. In contrast to unforeseeable events or 'crises' requiring an urgent response (such as an unforeseen attack or natural disasters), the fact of anticipation means that planning can be conducted with some systematic deliberation over periods from a week out to several months.

In Australia, the MAP consists of: Scoping (determining the context for the military problem to be addressed), Mission Analysis (defining the problem), Course of Action Development (determining options available to tackle the problem), Course of Action Analysis (testing the options against each other to determine which is best) and Decision (writing orders to enable plan implementation). Some of us have previously been involved in modelling and simulating such a process for the Australian Defence Force (Wong and Kalloniatis 2007). Zhang et al. (2001) have applied Coloured Petri Net modelling in the ADF context, and Dutch researchers (Grant et al., 2008) have explored process modelling of the OPP.

In the way we have explained the MAP, military planning is essentially structured decision making. There are limits to how far this can be represented as a sequential process. Military planning - as in many business organisations - dovetails into and out of strategy formation. For Mintzberg, the latter cannot be decomposed into sub-processes but is an "impenetrable black box" (1994: p331) that resides within formal planning stages 
of organisations. In the absence of a microscopic theory for human creativity and will (the two key dimensions of Command for Pigeau and McCann (2000)), the decomposition of planning into ever smaller components of activity at some point must terminate. Another limitation is that in its progress from problem formulation to solution, military planning can be a Wicked Problem (Rittel and Webber, 1973): planners may backtrack as problem formulation is recast in light of a partial solution (Wong and Kalloniatis, 2007, Kalloniatis and Macleod, 2010). The triggers for such backtracking are as much a consequence of creative spontaneity through deepening insight into a Wicked Problem (as emphasised by Mintzberg (1994), without using the Rittel and Webber concept) as on changing external conditions, such as when planning is concurrent with execution (Alberts and Hayes, 2007). There is thus limited value to modelling planning as an ordered sequence of steps with clearly demarcated purposes.

Contrastingly, in the present era the military forces of nations such as Australia, the USA, the UK and Canada, have been engaged in a number of ongoing campaigns lasting for a decade or more (Afghanistan, East Timor, the Solomon Islands). A form of long-cycle periodicity tends to develop around the planning of different phases of such operations, with periodic reviews of campaigns a common practice.

This coexistence of regularity and irregularity leads us to a Stochastic Cycles Concept (an elaboration of our previous Competing Cycles Concept (Kalloniatis et al., 2009)) for military planning. Staff work is dynamically distributed, and personnel are stressed according to interference between temporal stochastic cycles, each of which describes the distribution of increments of work leading to a specific product for a specific operation. The pattern of work reflects the MAP but is overlaid with stochasticity. Elementary tasks are activities of teams of people. These activities - which are not identified by any particular MAP step - are initiated according to statistical distributions.

\section{MODEL STRUCTURE AND DATA}

\subsection{Overview}

A critical aspect of the model design is that it be suitably placed to enable comparisons between 'as-is' and alternative 'to-be' staffing for the conduct of a fixed workload. Thus, the number of available staff has to be separated from the volume of work itself. Figure 1 shows how an intermediate layer provides the link between these for planning functions. More generally, such a decoupling is applicable to the full range of functions in a military operational headquarters running activities across multiple Areas of Operation (AOs). Australia's Headquarters Joint Operations Command, the UK's Permanent Joint Headquarters and the NZ Headquarters Joint Forces are examples of this structure.

\subsection{Types of planning}

Our hypothetical military headquarters is responsible for planning, monitoring and control in two separate geographical AOs: $\mathrm{AO} 1$ and $\mathrm{AO} 2$. This means that the overall Commander must prioritise the use of finite military resources across both AOs. The planners, who we model here, help to inform these priorities. The headquarters undertakes two types of planning: Deliberate Planning involves identification of likely options for possible future contingencies and developing high level generic strategies, while Immediate Planning is concerned with more specific, likely eventualities that naturally require more detailed plans. All planning is in the context of ongoing operations in parts of the two AOs. A plan may be a refinement or revision of plans for a current operation in progress (known as 'Branches' and 'Sequels' in military parlance). Alternatively, the planning may be for an entirely new operation in a new part of the AO. The planning may be

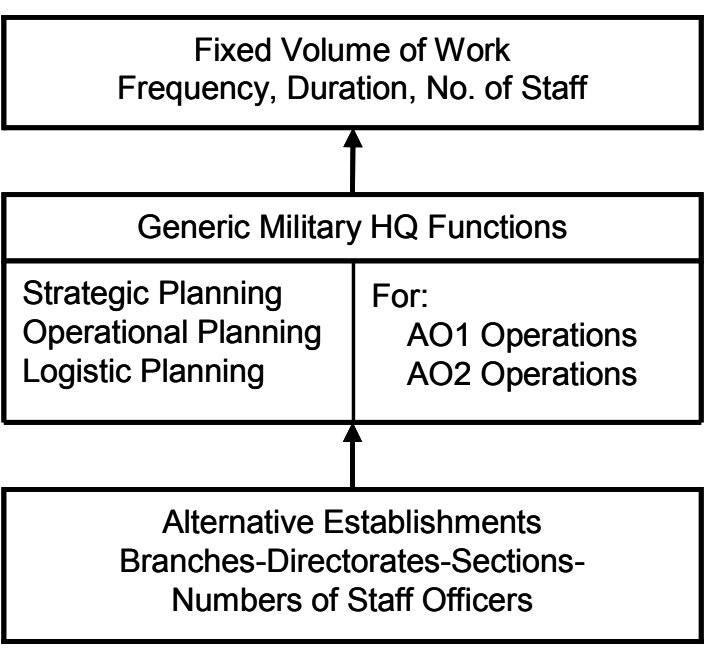

Figure 1. Model Conceptual Design led by one of two groups: Strategic Planners when the planning is Deliberate and Operational Planners when the planning is Immediate. However, for both types of planning, Deliberate and Immediate, both strategic and operational level considerations may be required. We also include Logistics Planners who develop the schedule of movement of materiel into and out of the AOs. These three areas call upon quite different skills: Strategic Planners are versed in grand-strategy, Operational Planners are expert in war-fighting ('Operational Art' (McKercher and Hennessy, 1996)) and Logistics Planners are specialists in maintaining a regular flow of equipment and resources. The model could easily include specialists in Intelligence, ICT Systems, Legal, 
Health, Personnel and Finance; we forego these for the sake of brevity. Thus, the hypothetical headquarters is structured with resources as given in Table 1.

A Chief of Staff would oversee the flow of work across all Branches, with the Commander in charge of all the relevant operations. Between the Chief of Staff and the specialist staff may occur more layers of intermediate officers whose work is not modelled here. The numbers in Table 1 may be a balance between overall scale of the operation (for example AO1 could be argued to be larger than AO2) and also the degree of strategic scope in the operation (activities in AO1 may be quite narrow in strategic scope compared with AO2). There is further rank

Table 1. Staff Structure of Hypothetical Headquarters structure within the staff, typically one or two teams of three or four Staff Officer Grades 2 or 3 (SO2/SO3) headed by a Staff Officer Grade 1 (SO1). However, in the model we present here these are equally available to conduct work.

\subsection{Structure of the data}

We use data based loosely on our work in real military headquarters, which is typically collected through a combination of interviews with staff officers and access to their diaries or Excel spreadsheets by which tasks are tracked. For the sake of brevity we provide an example of such source data in Table 2. Here time periods are year $(\mathrm{y})$, quarter $(\mathrm{q})$, month $(\mathrm{m})$, week $(\mathrm{w})$, day $(\mathrm{d})$ and hour (h). The six Staff Groups of Table 1 are the individual specialist sections of staff officers from which a number may be drawn for a planning activity. Frequency, varying from $3 / y-48 / y$, is the average number of times per year the distinct planning activities occur. The Window, from a week to a quarter, is the period of time within which the overall planning must be completed. The Quantity of Staff, varying from $1-4$, is the number of staff

Table 2. Hypothetical Data for AO1 Immediate Planning

\begin{tabular}{|l|c|c|c|}
\hline Activity & \multicolumn{3}{|c|}{ AO1 - NewOp - Immediate - } \\
& \multicolumn{3}{|c|}{ Plans Led } \\
\hline Staff Group & Strat AO1 & Plans AO1 & Log AO1 \\
\hline Frequency & $3 / y$ & $3 / y$ & $3 / y$ \\
\hline Window & $\mathrm{m}$ & $\mathrm{q}$ & $\mathrm{q}$ \\
\hline Qty of Staff & 2 & 1 & 3 \\
\hline Iterations & 4 & 20 & 9 \\
\hline Time/iteration & $8 \mathrm{~h}$ & $8 \mathrm{~h}$ & $8 \mathrm{~h}$ \\
\hline
\end{tabular}
from each Staff Group required for each planning iteration. Number of Iterations, which ranges from 2-20, is the number of steps of continuous activity within the Window into which the work required for a plan can reasonably be divided. Time per Iteration is the total uninterrupted time that the number of staff will be engaged in an iteration before moving to a different task. The duration of iterations ranges from 2 to 8 hours. We also model a one hour reading by staff of Situation Reports at the beginning of each workday, through which personnel maintain Situational Awareness.

The initiation for planning is triggered according to the data, using a uniform random distribution. If the events in the environment calling for military planning were truly uncorrelated then there would be an argument for using Poisson statistics. However, because events are in a geographic area broadly within which the military force is undertaking operations there is evidently some correlation. A uniform random distribution assumes as little as possible in light of the data. Planning activities are also staggered in time, consistent with the broad flow of the MAP or OPP. Strategic Planners commence work first. Operational Planners engage when the strategy is sufficiently developed to allow the Mission to be identified. When sufficiently concrete facts or assumptions have been made about the Mission and possible Courses of Action, Logistics Planners consider the scheduling of materiel flows. Many of these activities are observed to be concurrent, which is reflected here through phased stochastic triggering.

\subsection{Modelling tool}

We used IBM's WebSphere Business Modeler Advanced (Version 6.1.2) to construct executable models and to conduct simulations. While any Discrete Event Modelling tool (such as Arena) would suffice, the strengths of IBM's tool are its simple graphical interface for model construction and its ability to show animation of 
control flow and task activity while simulations are executing. The tool has an inbuilt capability to conduct some statistical analysis and to export data to Excel for further analysis, tabulation and graphing.

Processes here consist of an input, control flow, one or more elemental tasks, an output, decision and branch points, and data repositories. Elemental tasks can be specified in terms of the input conditions to be met before they commence execution, the resources they require, their duration, and the number and type of outputs they produce. Available resources take the form of roles and individuals. The distinction allows us to achieve the modular construct of Figure 1 directly: Tasks are performed by Role resources while Roles are fulfilled by Individual Resources. Thus, a change of staff numbers in examining various 'to-be' organisations is achieved by changes in the number of Individual Resources while keeping the rest of the model intact. Timers and calendars enable representation of recurring activities. The timers have two inputs: enable and suspend. In some cases we wish the timers to issue one and only one trigger - this is achieved by coupling the timer's trigger output back to its suspend input. Spreading the workload associated with an activity type (such as the example in Table 1) over the allowable window is achieved by a combination of fixed delays and timers to ensure that work elements are not triggered at night or on weekends (they may, however, commence or complete outside normal working hours because of backlogs).

\section{SIMULATION OUTPUT AND ANALYSIS}

\subsection{Manipulating the IBM Modeler's basic output and verification}

The IBM tool provides for analysis such as activity duration and resource usage. Resource usage analysis exposes how each resource is allocated to different activities across the simulation period, which of the resources suffer shortages and which activities are consequently delayed. The rows of data in the raw output, varying here from 9,000 to 25,000 in number for a 3 year simulation period, correspond to an individual staff member executing an elemental task in the model. Such raw model outputs are difficult to interpret because of their strict time order, and the interspersing of different activity elements. We extract for each task iteration the number of staff and duration in hours to calculate total hours of work for that day, together with the hours of work per individual in each Staff Group for each day. Using Excel formulae for pattern matching, extraction of substrings, and conditional IF and SUM functions we verify that the total hours across Staff Groups for each activity type correspond to the figures calculated from the corresponding source data. The total hours across activity types for each Staff Group are similarly confirmed. In preparing the following plots, the overall workload is divided into totals for each activity type for each Staff Group. This allows a finer level of verification, in which simulation output totals agreed with the source data.

\subsection{Daily fluctuations of work per Staff Group}

To gain an impression of how diverse the daily workload could be for a particular Staff Group, the output is disaggregated in Excel into individual activities. Work elements are first sorted by date and time of initiation. The durations of multiple work elements for a given activity within each work day are summed and combined into a single spreadsheet row for each day, giving the number of hours for each type of activity. Sets of these rows covering representative date ranges are then plotted for the Staff Groups, as shown in Figure 2 for three AO1 Staff Groups. This figure shows a number of features. Dominant for all three Groups is Immediate Planning for Current Operations (red bars). The workload is volatile and fragmented, with up to four distinct activities on some days. The larger gaps between bars indicate weekends. However, logistics planners (lower panel) occasionally have work flow onto weekends. We can also observe the broad impact of the process: Immediate Planning for a new operation in AO1 - green bars - has been initiated early in the window (upper panel) with Strategic Planners developing the initial strategy. The work has flowed to the operational planners in the middle part of the time window (middle panel) and logistics input occurs toward the end of the window (lower panel). However, all this work is interspersed with other ongoing tasks.

\subsection{Testing scenarios}

To demonstrate the utility of the model for decisions about staff distribution, we now aggregate across all tasks falling to a Staff Group to calculate the average work hours per person in the Staff Group. Because of the volatile nature of the fluctuations (itself amplified by the use of uniform random distributions for triggering planning initiation), there is no optimal window on the simulation that avoids initiation/finalisation transients and other statistical vagaries. The means and standard deviation for workloads of the various Staff Groups are calculated using a 2.5 year period, starting after 3 months into a 3 year simulation and finishing 3 months before the end. Obviously for input to a decision-maker such a process should be undertaken for a longer run or over a large number of separate simulations in order to identify appropriately comparable periods (given that fluctuations in average workloads persist over extended intervals). 
Estimates of staff average working hours with the 'as-is' model are given in Figure 3; the error bars are standard deviations for the working hour distributions of $\sim 600$ work days in the 2.5 year simulation period. The large standard deviations reflect the volatility in working hours evident in Figure 2. (Standard errors for the estimates are approximately $1 / \sqrt{ } 600$ smaller as a result of the sample size.) We observe a very heavy average work-day for logistics planners in AO1 while all others are broadly consistent, though operational planners have evidently lighter days. This is a simple property of the number of staff available. We note that we are not modelling meal times (which may be taken at the desk in the heavily loaded Staff Groups or taken in the Mess by those with more appropriate work days), physical training (which may be taken during the work day if time is available), and many personal administrative tasks (such as personal banking and medical appointments), or corporate activities such as travel acquittal or training.

We show three scenarios for mitigating the heavy workloads of the AO1 logistics planners: to-be- 1 = pooling of the AO1 and $\mathrm{AO} 2$ logistics planning staffs; to-be- 2 = one less AO1 operational planner for AO1 and one extra AO1 logistics planner, but separate AO1 and $\mathrm{AO} 2$ staffs. While the first scenario could be implemented by the logistics planning Branch Head within a posting cycle, the second could be made only from one cycle to the next: it is impractical for an operational planner to become expert in logistics aspects. The new average loads are indicated by 'to-be-1' and 'to-be-2' respectively in Figure 3. We see that pooling the logistics resources has some effect on

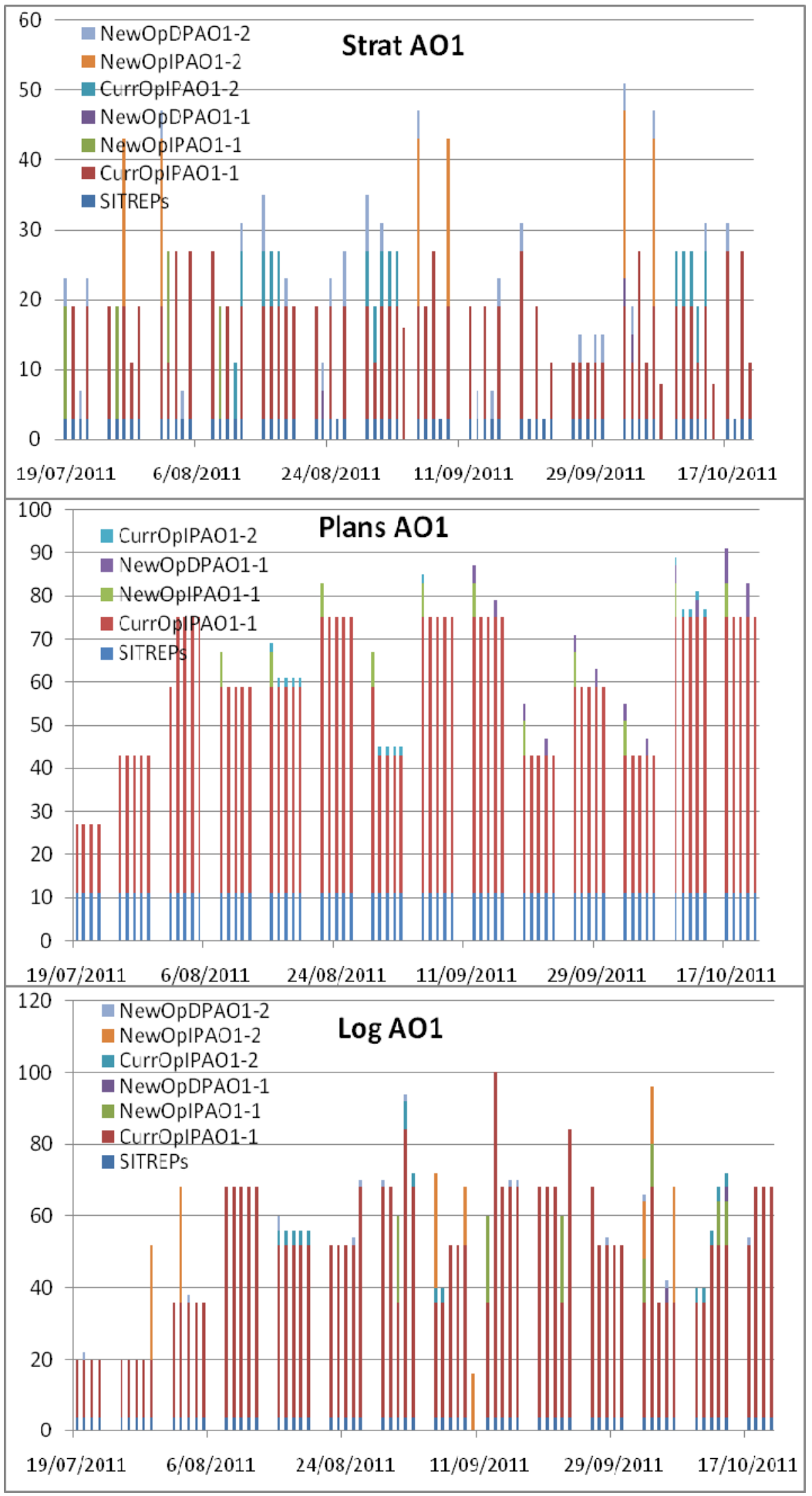

Figure 2. Workload Activity by Task and Day balancing the workload across Staff Groups. An extra logistics planner helps balance workloads better, without great impact on the operational planners. Kolmogorov-Smirnov statistics (Kirkman, 1996) for the asis, to-be- 1 and to-be- 2 simulations confirm that the distributions of staff workloads under these scenarios differ at better than the $\mathrm{p}=0.001$ level. 


\section{DISCUSSION AND CONCLUSIONS}

Our Stochastic Cycles approach to modelling headquarters planning activities acknowledges that creative organisational work is highly dynamical and follows linear processes only loosely, and is thus a step closer to representing the daily mix of concurrent planning activities than earlier approaches. The new approach is more data intensive than our previous Competing Cycles representation of headquarters Monitoring and Control. However, by avoiding individual interactions it requires less data than social network or agent representations for similarly sized organisations.

Using data easily represented in Excel provides for its accumulation by real staff and for coarse steadystate analysis within spreadsheets. Fewer constraints on data collection allow greater fidelity in this model, such as distinguishing individual planning stages, breaking work down to sub-teams, task shedding and the introduction of task priorities. This approach is as close as one can get with manageable data before 'agent modelling' where primitive models of decisionmaking influence workload distribution - becomes viable. Though not suited for questions about plan quality (which, arguably, cannot be tested in isolation from plan execution) this new model of military planning can help answer questions about staff resource

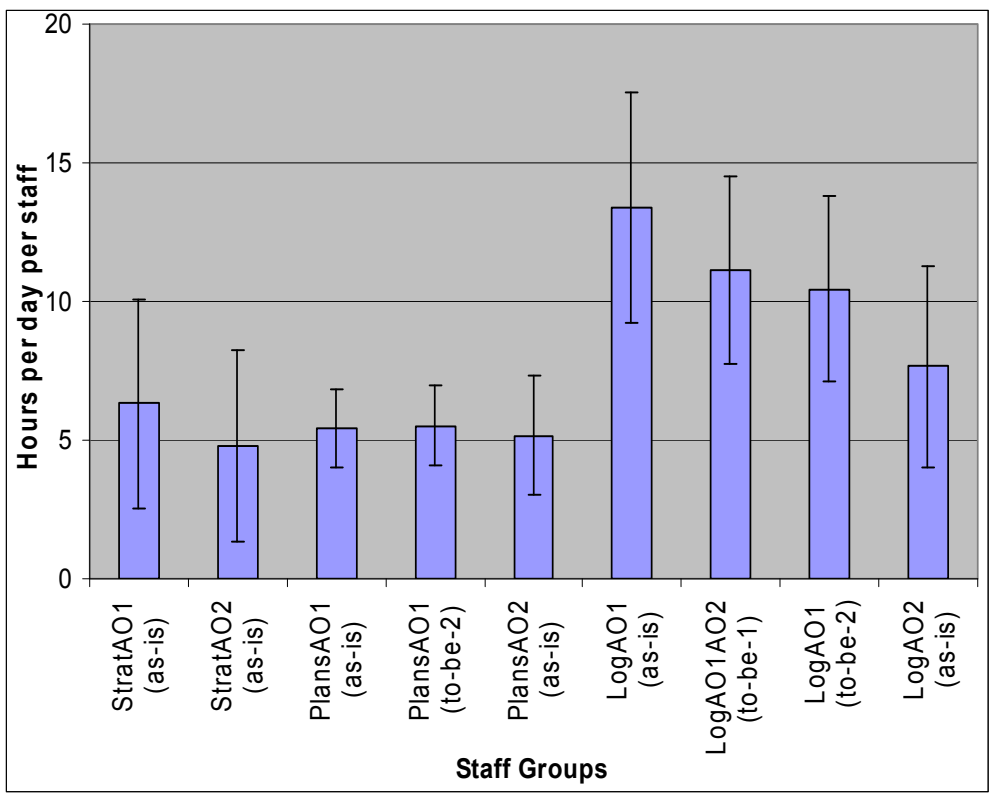

Figure 3. Average Work by Staff Group and Model distribution, taking into account both the regular and irregular nature of organisational work.

\section{REFERENCES}

Alberts, D.S., and Hayes, R.E. (2007), Planning: Complex Endeavours, CCRP, USA.

Aguilar-Savén, R.S. (2004), Business process modelling: review and framework, Int. J. Production Economics, 90, 129-149.

Grant, T., Essens, P., and van der Kleij, R. (2008), Reducing operational planning cycle time using BPR and concurrent engineering, 13th International Command and Control Research and Technology Symposium (ICCRTS), Seattle, USA, 17-19 June 2008, Conference CD.

Guitouni, A., Wheaton, K., and Wood, D. (2006), An essay to characterise models of the military decisionmaking process, $11^{\text {th }}$ International Command and Control Research and Technology Symposium (ICCRTS), Cambridge, UK, 26-28 September 2006, Conference CD.

Kalloniatis, A.C., Macleod, I.D.G., and La, P. (2009), Process versus battle-rhythm: modelling operational Command and Control, $18^{\text {th }}$ World IMACS/ MODSIM Congress, Cairns, Australia, 13-17 July 2009.

Kirkman, T.W. (1996), Statistics to Use. http://www.physics.csbsju.edu/stats (accessed 7 September 2011).

McKercher, B.J.C., and Hennessy, M.A. (1996), The operational art: developments in the theories of war, Praeger, Westport, Connecticut.

Mintzberg, H. (1994), The Rise and Fall of Strategic Planning, Free Press, NY.

Pigeau, R., and McCann, C. (2000), Redefining Command and Control, Chapter 12 (pp163-184) in The Human in Command, Pigeau, R. and C. McCann (Eds.), Kluwer Academic/Plenum.

Rittel, H.W.J., and Webber, M.M. (1973), Dilemmas in a general theory of planning, Policy Sciences, 4, $155-169$.

Wong, P., and Kalloniatis, A. (2007), Application of business process modelling to military organisations, SimTechT Conference, Brisbane, Australia, June 2007.

Zhang, L, Kristensen, L.M., Falzon, L., Davies, M., Mitchell, B., and Billington, J. (2001), Model-based operational planning using coloured Petri nets. $6^{\text {th }}$ International Command and Control Research and Technology Symposium (ICCRTS), Annapolis, USA, 19-21 June 2001, Conference CD. 